\title{
Local cerebral blood perfusion correlates with nerve fibre integrity in transient ischemic attack patients with middle cerebral artery stenosis: a pilot study
}

\author{
Jiang $\mathrm{Wu}^{1}$, Ping $\mathrm{Liu}^{2}$, Jie $\mathrm{Lei}^{3}$, Jia Liu ${ }^{1,4}$, Hong-Liang Zhang ${ }^{1,4 *}$ \\ ${ }^{1}$ Department of Neurology, First Hospital of Jilin University, Changchun, China \\ ${ }^{2}$ Department of Orthopaedics, Fourth Hospital of Jilin University, Changchun, China \\ ${ }^{3}$ Department of Paediatrics, First Hospital of Jilin University, Changchun, China \\ ${ }^{4}$ Department of Neurobiology, Care Sciences and Society, Karolinska Institute, Stockholm, Sweden; \\ *Corresponding Author: Hongliang.Zhang@ki.se
}

Received 21 January 2010; revised 23 February 2010; accepted 26 February 2010.

\begin{abstract}
Recent advances in neuroimaging contribute a lot to the accurate diagnosis and evaluation of cerebrovascular diseases. To explore the relationship among blood perfusion, metabolism and brain structure integrity, 6 Chinese transient ischemic attack (TIA) patients with middle cerebral artery (MCA) stenosis were examined by xenon-enhanced computed tomography (Xe-CT), magnetic resonance spectroscopy (MRS) and diffusion tensor imaging (DTI) to compare cerebral blood flow (CBF) values, (choline + creatine)/ $\mathrm{N}$-acetyl aspartate [(Cho $+\mathrm{Cr}) / \mathrm{NAA}]$ values and fractional anisotropy (FA) values in the MCA territory. Our results showed that CBF values significantly decreased in the ipsilateral basal ganglion regions in all 5 cases with unilateral MCA stenosis, with a corresponding decrease of FA values in the same region. In conclusion, decreased blood perfusion may indicate nerve fibre damage in the dominating regions of stenosed arteries.
\end{abstract}

Keywords: Transient Ischemic Attack;

Magnetic Resonance Imaging;

Xenon-Enhanced Computed Tomography

\section{INTRODUCTION}

Knowledge on recurrence, optimal evaluation and effecttive prevention strategy of transient ischemic attack (TIA) is still lacking. An increasing body of evidence has revealed that TIA in Chinese population is more related to intracranial artery stenosis [1]. Recent advances in neuroimaging contribute a lot to the accurate diagnosis and evaluation of TIA and cerebrovascular stenosis. To address the relation between blood perfusion, structure integrity and metabolism in the brain, we enrolled Chinese TIA patients with middle cerebral artery (MCA) stenosis to compare cerebral blood flow (CBF), (choline + creatine $) / \mathrm{N}$-acetyl aspartate $[(\mathrm{Cho}+\mathrm{Cr}) / \mathrm{NAA}]$ and fractional anisotropy (FA) values of MCA territory measure by xenon-enhanced computed tomography $(\mathrm{Xe}-\mathrm{CT})$ and magnetic resonance imaging (MRI).

\section{SUBJECTS AND METHODS}

A total of 6 consecutive TIA patients with MCA stenosis detected by TCD and confirmed by MRA, were enrolled to undertake Xe-CT, MRS and DTI examinations 3 days after the most recent attack in the department of radiology from January to June in 2009. TIA was as previously defined [2]. Study protocol and informed consent were obtained from all participants in the study, which was conducted in accordance with institutional guidelines. Patients with lacunar infarction in the basal ganglion of CT or MRI were excluded from this research

All patients had a full clinical assessment, including electrocardiogram (ECG), carotid duplex Doppler Ultrasonography, and mini mental status examination (MMSE) and National Institutes of Health Stroke Scale (NIHSS). American Nicolet TC8080 TCD machine, German Siemens MAGNETOM Avanto 1.5 T MR machine was used to perform MRI, MRA, DTI and MRS examination, and American Diversified Diagnostic Products Xe-CT CBF system with German Simens PLUS4 CT were used to perform Xe-CT examination.

MRI Protocol: MRI scanning, including conventional MR images, including T2-weighted, T1-weighted, and 
fluid-attenuated inversion recovery (FLAIR) images, MRA, MRS and DTI were performed with the use of a clinical $1.5 \mathrm{~T}$ whole-body MR system Siemens MAGNETOM Avanto with a conventional gradient system (Magnetom Vision, Siemens Medical System). Parameters are as follows: TSE/T1WI: TR $400 \mathrm{~ms}$, TE $7.8 \mathrm{~ms}$; TSE/T2WI: TR $3250 \mathrm{~ms}$, TE $99 \mathrm{~ms}$; Time-of-flight (TOF)-MRA, scanning time 6'01", TR $25 \mathrm{~ms}$, TE $4.6 \mathrm{~ms}$, thickness $0.9 \mathrm{~mm}$, band width $85 \mathrm{~Hz}$; $\operatorname{MRS}\left({ }^{1} \mathrm{H}\right)$ : twodimensional MRS of basal ganglion, scanning time 7'12", TR $1500 \mathrm{~ms}$, TE $135 \mathrm{~ms}$, band width $35 \mathrm{~Hz}$; DTI: TR $3900 \mathrm{~ms}$, TE $76 \mathrm{~ms}$; DTI: TR $3900 \mathrm{~ms}$, TE $76 \mathrm{~ms}$, matrix $64 \times 64$, in-plane resolution was $1.875 \times 1.875 \mathrm{~mm}$.

Xe-CT protocol: Xe-CT studies used a 28 to $33 \%$ concentration of medical-grade xenon gas mixed with $\mathrm{O}_{2}$. At the start of each study, a face mask was used to deliver the mixed gas and the patient was closely monitored. The procedure used within the DDPI system involved obtaining two baseline scans before Xe inhalation and six scans during Xe inhalation for each of four axial planes of the brain, each $20 \mathrm{~mm}$ thick. CT scanning was performed at intervals of $1 \mathrm{~min}$ at the basal ganglial level. The resulting image was shown as a CBF map, on which $\mathrm{CBF}$ values could be extracted by placing regions of interest (ROIs) on corresponding brain tissue.

The results of TCD and MRA were independent of the patients' clinical manifestations, given by professional TCD and MRI technicians. And the (Cho $+\mathrm{Cr}) / \mathrm{NAA}$ values, $\mathrm{FA}$ values and $\mathrm{CBF}$ values of the basal ganglion regions were measured and calculated by three respecttive professional radiological physicians blinded to the clinical data of the subjects. Results are presented as mean $\pm \mathrm{SD}$. The data of FA and $(\mathrm{Cho}+\mathrm{Cr}) / \mathrm{NAA}$ values were analysed with nonparametric analysis, Wilcoxon Signed Ranks Test by software of SPSS 11.5 (for Windows OS). And CBF values were analysed with PEMS 3.0 to compare means of two samples. A probability value of $<0.05$ was considered significant.

\section{RESULTS}

All 6 subjects were male, aged $55.7 \pm 10.3$; ECG, carotid duplex Doppler ultrasonography, MMSE and NIHSS showed negative results. Average CBF values in bilateral ROIs were different, with the maximum difference of $12.2 \mathrm{ml} /(100 \mathrm{~g} \cdot \mathrm{min})$, and the minimum of $1.5 \mathrm{ml} /(100$ $\mathrm{g} \cdot \mathrm{min})$; CBF values significantly decreased in the ipsilateral basal ganglion regions of stenosed MCA in all 5 cases with unilateral MCA stenosis $(\mathrm{P}<0.05)$. (Table 1$)$ FA values in stenosed MCA territory were significantly lower than those in the contra-lateral counterparts in 5 subjects with unilateral MCA stenosis. There was no
Table 1. CBF values measured by $\mathrm{Xe}-\mathrm{CT}[\mathrm{ml} /(100 \mathrm{~g} \cdot \mathrm{min})]$.

\begin{tabular}{cccc}
\hline Subject No. & Ipsilateral & Contralateral & $\boldsymbol{p}$ value \\
\hline 1 & $45.6 \pm 17.0$ & $51.9 \pm 19.9$ & 0.0000 \\
2 & $45.2 \pm 17.1$ & $56.7 \pm 20.9$ & 0.0000 \\
3 & $59.0 \pm 20.3$ & $63.4 \pm 24.8$ & 0.0000 \\
4 & $32.9 \pm 17.7$ & $40.0 \pm 14.7$ & 0.0003 \\
5 & $52.7 \pm 17.5$ & $64.9 \pm 23.0$ & 0.0000 \\
$* 6$ & $56.1 \pm 18.4$ & $57.6 \pm 23.3$ & 0.1270 \\
\hline
\end{tabular}

* Bilateral MCA stenosis.

difference when comparing $(\mathrm{Cho}+\mathrm{Cr}) / \mathrm{NAA}$ values of bilateral MCA territory.

\section{REPRESENTATIVE CASE}

A 53-years old male with a history of untreated hypertension and diabetes mellitus, presented ictal left hemiparesis for the first time. Dizziness, vertigo, nausea and dysarthria were absent. TCD suggested a reduced blood flow velocity of right MCA. Stenosed right MCA was confirmed by MRA. Xe-CT showed decreased CBF in the right basal ganglion, with $\mathrm{CBF}$ of $45.6 \pm 17.0 \mathrm{ml} /(100$ $\mathrm{g} \cdot \mathrm{min})$ compared with $51.9 \pm 19.9 \mathrm{ml} /(100 \mathrm{~g} \cdot \mathrm{min})$ in the right counterpart $(p<0.05)$ FA values in stenosed MCA territory were significantly lower than the counterpart. $(p$ $<0.05)$ There was no significant difference between bilateral $($ Cho $+\mathrm{Cr}) /$ NAA values $(p>0.05)$ (Figure 1).

\section{DISCUSSION}

$\mathrm{Xe}-\mathrm{CT}$ is a quantitative method of $\mathrm{CBF}$ analysis, and multiple studies have validated the accuracy of CBF values obtained with Xe-CT [3]. MRS can evaluate the metabolism of brain tissues in vivo [4]. Abnormalities of neuronal structures lead to reductions in NAA quantity [5]. An increase in the Cho peak is associated with conditions such as demyelinating disease and brain tumors [6]. Also alterations in the NAA/Cho ratio have been reported with findings suggestive of neuronal damage caused by neuronal disorders [7]. In theory, the chronic or acute ischemia of brain tissue may result in the changes of $(\mathrm{Cho}+\mathrm{Cr}) / \mathrm{NAA}$ due to axonal degeneration or demyelination secondary to artery stenosis, while in the present study, no significant changes in $(\mathrm{Cho}+\mathrm{Cr}) /$ NAA value could be seen. The similar results can be seen in a previous MRS study in TIA without stenosed cerebral artery [8]. One reason may be argued that although there is a reduction of CBF in the territory of 


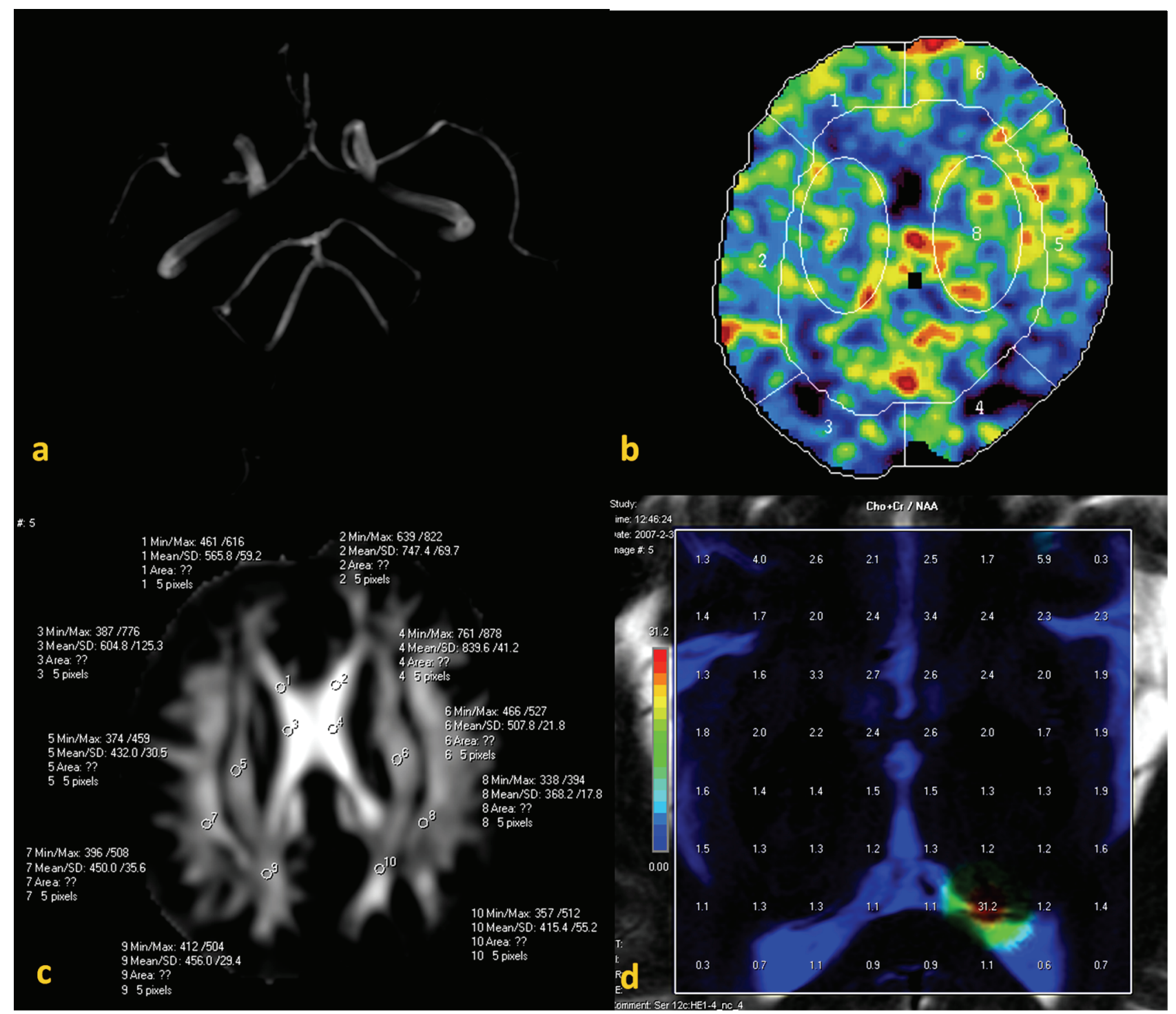

Figure 1. (a) MRA showed stenosed right MCA in the representative case; (b) Xe-CT showed decreased CBF in the right basal ganglion, with CBF of $45.6 \pm 17.0 \mathrm{ml} /(100 \mathrm{~g} \cdot \mathrm{min})$ compared with $51.9 \pm 19.9 \mathrm{ml} /(100 \mathrm{~g} \cdot \mathrm{min})$ in the right counterpart. $(p=0.000)$; (c) DTI showed a decrease of FA values in stenosed MCA territory. $(p=0.008)$; (d) MRS showed no significant difference in bilateral ROIs. $(p=0.866)$.

stenosed MCA compared with the contralateral counterpart, the relative ischemia of the brain tissue may not necessarily result in a significant metabolic change. DTI may be used to map and characterize the three-dimensional diffusion of water as a function of spatial location. Chronic ischemia of brain is postulated to possibly cause demyelination of white matter, which can result in the reduction of FA values. And our data confirmed such a hypothesis.

Although only 6 patients contributed to this study, a preliminary conclusion of the present study may be drawn: local changes of $\mathrm{CBF}$ may be used to estimate the integrity of nerve fibres in TIA patients with stenosed MCA.

As regards the limitation of our study, the primary one is the small number of the subjects; Secondly, although blind method was used, there still involved subjective deflection in the measurement and calculation of CSF, $\mathrm{FA}$ value and $(\mathrm{Cho}+\mathrm{Cr}) / \mathrm{NAA}$ values; Thirdly, the relatively low field intensity of 1.5 T MRI is another weakness in performing MRS and DTI examination; Also, lactic acid, which can directly reflect the degree of hypoperfusion and hypoxemia, was not detected.

In summary, the combined use of CT and MR tech- 
niques to quantitatively assess blood perfusion, metabolism and water molecule diffusion may be a first step towards accurate diagnosis and evaluation of TIA and intracranial artery stenoses and may in the future allow effective prevention of stroke and monitoring of treatment strategy.

\section{ACKNOWLEDGEMENTS}

This study was supported by the China Scholarship Council. We thank Hongwei Zhou, Jing Wang and Jing $\mathrm{Xu}$, for kindly help in subject screening.

\section{REFERENCES}

[1] Li, D., Wang, M.L., Li, S.M. and Ling, F. (2008) Distribution and risk factors of steno-occlusive lesions in patients with ischemic cerebrovascular disease. National Medical Journal of China, 88(17), 1158-1162.

[2] Albers, G.W., Caplan, L.R., Easton, J.D., Fayad, P.B., Mohr, J.P., Saver, J.L., Sherman, D.G. and TIA Working Group (2002) Transient ischemic attack-proposal for a new definition. New England Journal of Medicine, 347 (21), 1713-1716.

[3] Latchaw, R.E., Yonas, H., Hunter, G.J., Yuh, W.T., Ueda, T., Sorensen, A.G., Sunshine, J.L., Biller, J., Wechsler, L., Higashida, R., Hademenos, G. and Council on Cardio- vascular Radiology of the American Heart Association (2003) Guidelines and recommendations for perfusion imaging in cerebral ischemia: a scientific statement for healthcare professionals by the writing group on perfusion imaging, from the Council on Cardiovascular Radiology of the American Heart Association. Stroke, 34(4), 1804-1104.

[4] Alkan, A., Sarac, K., Kutlu, R., Yakinci, C., Sigirci, A., Aslan, M. and Baysal, T. (2003) Early and late-state subacute sclerosing panencephalitis: chemical shift imaging and single-voxel MR spectroscopy. American Journal of Neuroradiology, 24(3), 501-506.

[5] Cecil, K.M. and Jones, B.V. (2001) Magnetic resonance spectroscopy of the pediatric brain. Top Magn Reson Imaging, 12(6), 435-452.

[6] Irwan, R., Sijens, P.E., Potze, J.H. and Oudkerk, M. (2005) Correlation of proton MR spectroscopy and diffusion tensor brain MR imaging. Magn Reson Imaging 23(8), 851-858.

[7] Kadota, T., Horinouchi, T. and Kuroda C. (2001) Development and aging of the cerebrum: assessment with proton MR spectroscopy. American Journal of Neuroradiology, 22(1), 128-135.

[8] Giroud, M., Walker, P., Guy, F., Lemesle, M., Lalande, A., Baudouin, N., Martin, D., Couveur, G. and Brunotte, F. (1999) Cerebral metabolism after transient ischemic attack. A 1H MR spectroscopy study. Neurological Research, 21(6), 563-565. 